\title{
Emergent Semantics: Towards Self-Organizing Scientific Metadata
}

\author{
Bill Howe, Kuldeep Tanna, Paul Turner, and David Maier \\ OGI School of Science \& Engineering at \\ Oregon Health \& Science University \\ Beaverton, Oregon \\ \{bill,kuldeep,maier\}@cse.ogi.edu, pturner@ccalmr.ogi.edu
}

\begin{abstract}
Tasked with designing a metadata management system for a large scientific data repository, we find that the customary database application development procedure exhibits several disadvantages in this environment. Data cannot be accessed until the system is fully designed and implemented, specialized data modeling skills are required to design an appropriate schema, and once designed, such schemas are intolerant of change. We minimize setup and maintenance costs by automating the database design, data load, and data transformation tasks. Data creators are responsible only for extracting data from heterogeneous sources according to a simple RDF-based data model. The system then loads the data into a generic RDBMS schema. Additional grouping structures to support query formulation and processing are discovered by the system or defined by the users via a web interface. Discovered and imposed structures constitute emergent semantics for otherwise disorganized information.
\end{abstract}

\section{Introduction}

When a group of environmental scientists requested our help designing a metadata management solution for their scientific data repository, we speculated that the database community's flagship technology, relational database management systems, would solve the problem neatly. We changed our assessment after further investigation, judging that the high cost of database deployment and maintenance made the likelihood of adoption rather low. As an alternative, we present a metadata collection, organization, and query architecture that lowers the cost of entry by reordering steps in the database design methodology. Metadata is gathered and loaded immediately using a simple RDF-based data model. After the data is loaded, they are partitioned according to their signature, which is inferred by the system. Additional grouping structures (views) can be defined by users through a web interface. Organizing data via signatures results in a form of schema that facilitates query expression and allows efficient query evaluation. By performing schema design semi-automatically only after the data is loaded, we sidestep the primary obstacles to database adoption in a scientific environment. Additionally, the schema structures we discover can be adapted to changes in the metadata stream, avoiding schema evolution problem. 
The context for our work is the CORIE Environmental Observation and Forecasting System [4], designed to support study of the physical processes of the Columbia River estuary. The CORIE system both measures and simulates the physical properties of the estuary, generating 5GB of data and over 20,000 data products and subproducts daily, including visualizations, aggregated results and derived datasets. The data products are consumed for many purposes, including salmon habitability studies and environmental impact assessments. The number of files in the repository is around 6 million and growing by tens of thousands a day. The existing repository is organized as a collection of directory structures on a Linux filesystem. Descriptions for these files are found encoded in the file name, in the content of the file itself, or in accompanying files.

For example, the data products generated from the simulation outputs have descriptive information encoded within their filenames. Figure 1 shows an example of descriptions found in and around the target file.

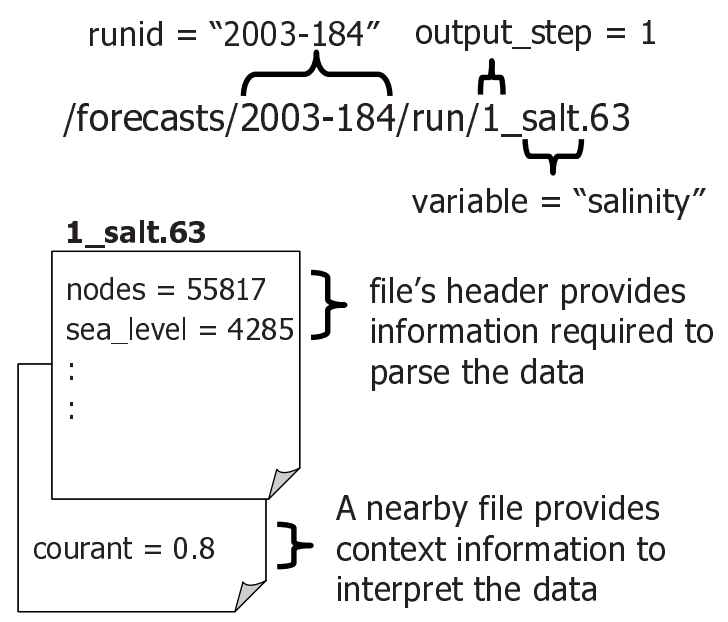

Fig. 1. Metadata encoded in file names, headers, paths, "nearby" files.

The data repository is exhibiting growth in both the number and types of files stored. We have encountered at least the following kinds of files:

- Observation data downloaded from field sensors.

- Simulation inputs downloaded from external servers, in varying stages of pre-processing.

- Simulation parameter files.

- Simulation control information such as logs, status flags, and saved checkpoints.

- Raw simulation outputs in multiple formats.

- Derived data products including reduced datasets, images, visualizations, reports, and aggregations of multiple datasets. 
Additionally, there is a growing need for access to these data by people other than the data creators; the heterogeneity of the sources of metadata across these file types is an impediment.

To support general data access for non-expert users through these scattered metadata, we describe two alternatives. Our initial plan was to build a straightforward database application. However, there was a possibility that such a system would be difficult to deploy and maintain without considerable ongoing help from us. After further thought, we aimed higher: a system for automatically and adaptively organizing metadata.

\subsection{The "Obvious" Solution}

Our first thought was to define a schema to house the metadata in an RDBMS. Next, data creators populate the schema by inserting a tuple for every file in the repository. Then, a database application is constructed and tested for searching the database.

There are several disadvantages to this approach. The data creators are not necessarily data modelers; schema design requires somewhat specialized skills. Even proficient data modelers would have trouble: The schema should be tuned to declared use cases, but since new users are coming online continuously, not all use cases are known at design time. We could undertake the modeling ourselves, but the effort would still require a lot of interaction with the scientists, who do not have much time to spare.

Data loading is also problematic; we cannot ask the scientists to manually enter 6 million tuples. Portions of the data-loading task can be automated since the data creators are often proficient programmers, but their knowledge does not necessarily include RDBMS languages or APIs. Data loading constitutes a significant up-front cost to this approach which must be paid before any benefit is realized, so it is difficult to persuade the scientists to diligently record metadata during the interim. Accurate test data with which to proof the system is also needed but has an even lower priority.

Once the data is loaded, the schema may be difficult to validate since the database is free to diverge from what is encoded in the filenames, file content, etc. Database APIs and a specialized schema constitute a "wide" interface between data creators and metadata managers: Small requirements changes can result in significant interface changes. A question of responsibility also arises: if a new metadata field is required, who must update the database and possibly modify or reload data?

\subsection{An Alternative}

Since the metadata sources may change frequently, those responsible for the changes should also be responsible for their extraction. If we perform the extraction, we are obligated to maintain the code even as the underlying requirements and metadata encodings change. If the scientists perform the database load, they 
will be responsible for maintaining the interface as the schema undergoes revision. We made the observation that decoupling metadata extraction from the rest of the system was important for robustness.

In our approach, the interface between metadata extraction and database loading is as narrow as possible. Data creators extract metadata "descriptors" and deliver them through a very simple data model based on RDF. Descriptors are extracted using collection scripts written by the data creators (or others) in their choice of programming language. These descriptors are loaded as-is into a generic RDBMS schema. Generic schemas are difficult to query and browse, and usually exhibit poor performance. We therefore partition the data into groups according to patterns found in the metadata. These groups act as a schema to expose structure, simplify query expression, enable browsing, and improve response time. Additional groupings of data can be defined by users through a web interface providing a personalized view of the repository.

This approach offers several advantages. A narrower interface between metadata managers and data creators is valuable: To accommodate changing metadata requirements, simply update the collection scripts and the system reacts appropriately. Metadata can be harvested and exploited prior to finalizing a schema, delivering benefits much sooner. A single, generic metadata delivery interface, controlled by collection scripts, can both load new data and update existing data. Since the schema is built dynamically, new views can be easily defined to support new users or new tasks. We use the term emergent semantics to describe the paradigm of harvesting data first and organizing it second. By organizing and publishing the gathered metadata back to users, they retain responsibility and control over validation and adjustments. The collection scripts used to gather metadata can be modified and re-executed to update the database.

Several challenges must be overcome to realize these benefits. Queries over a generic RDF schema are expensive and difficult to express. Techniques for identifying patterns in the metadata stream must be devised. Once patterns in the metadata are found, we must convert them into structures (signatures and signature extetnts) to facilitate query expression and processing. Users may also wish to impose their own structure on the data. As new users and new use cases are introduced, requirements may change. If the metadata stream changes, can the previously found structures adapt? In some cases, users may wish to promote previously found structures into hard constraints, rejecting non-conforming metadata. This paper presents an architecture that takes initial steps towards resolving these issues.

\section{Harvesting Metadata}

To extract metadata from scattered sources, we rely on collection scripts to assign $\langle$ property, value $\rangle$ pairs to each file. The output of collection scripts are then interpreted as $\langle$ subject, property, object $\rangle$ triples. In our application, collection scripts are written primarily, but not exclusively, by the environmental scientists. 
They are proficient programmers and are most familiar with the file description encoding schemes at the sources.

Collection scripts may be written in any language. To fire the collection scripts and harvest the metadata we provide a program harvest. The harvest program accepts a set of applicability rules and a target directory as input, and produces 〈file, property, value〉 triples for each file in the directory. An applicability rule consists of a regular expression, the path to a collection script, and a path to an interpreter for the collection script, if necessary. The harvest program recursively walks the target directory, testing the rules for each file. A rule is activated if its regular expression accepts the string formed by the path and file name being tested. For each activated rule, the program executes the appropriate collection script. Multiple rules may fire for the same file, and the same collection script might be triggered by multiple rules.

For the file in Figure 1, a simple rule matching the ".63" extension triggers a collection script that emits triples from the file name (e.g., <1_salt.63, variable, salinity>), from the content (e.g., <1_salt.63, nodes, 55817>), and from nearby files (<1_salt.63, implicitness, 0.8>). Alternatively, these three sources could have been accessed by three separate collection scripts (requiring three rules), all triggered by the same regular expression. Rules give collection script writers significant flexibility with regard to software design. For example, these three collection scripts may be written by different authors, at different locations, with different metadata requirements.

Each script is expected to accept one command line argument, a file path, and call a function Assert (property, value, type) for each metadata item it wishes to record for the file. The Assert function is provided by the system for a few languages (Perl, Python, C). If scripts are written in a language for which the assert function has not been defined, the script may simply emit the property, value, and type arguments to standard out in a comma-delimited format.

Initiating a separate process for each script execution is prohibitively expensive, as there are around 20,000 files per run and a large repository of existing runs. To improve performance, the harvest program uses embedded interpreters for the two most popular languages, Perl and Python. For simplicity, the users still write their code as if it is to be run from the command line; the harvest program wraps each script in a virtual environment and executes it in the same address space.

The Assert function, called from within a collection script, emits a triple through temporary files or stdout. The stream of triples produced by a series of calls made by amultiple collection scripts is then delivered to a relational database for analysis, as described in Section 4.

\section{$3 \quad$ Modeling Metadata}

To interpret extracted metadata, we must choose a data model to capture the descriptions. Rich data models that can capture complex relationships, constraints, and operations allow the same real world concept to be modeled in 
many different ways. To choose an appropriate translation into the data model's structures, modeling experts meet with domain experts, clarify the details, and construct a prototype. This offline coordination is expensive in terms of time required, and usually involves several iterations. Other confounding issues impede the data modeling efforts: dirty data, redundancy, ambiguity. Finally, if changes are expected to be frequent, this design process might be repeated several times.

We sidestep these issues at this stage by adopting a very simple data model. Very little transformation need be performed and very few initial modeling decisions need be made to get the metadata recorded and queryable. Once the descriptions are in a uniform, machine-readable format, richer modeling features can be applied. Figure 2 contrasts the manual effort required by the two approaches. In Figure 2, the term "Signature Extent" refers to the grouping structures inferred by the system and will be explained in section 4.2 .

To accommodate the heterogeneity of the data sources and simplify the scientists' collection scripts, we adopt a data model based on the Resource Description Framework (RDF) [7] consisting of $\langle$ subject, property, object $\rangle$ triples. We sacrifice expressive power for simplicity and uniformity: Simple facts can be recorded immediately without regard to overall structure.

Of course, the additional expressive power of richer data models is important for query formulation and processing. To recover these benefits, we must derive relationships encoded in the $\langle$ subject,property, object $\rangle$ triples. For example, if several files all have the same set of properties, we can group the files together to simplify queries and improve their performance.

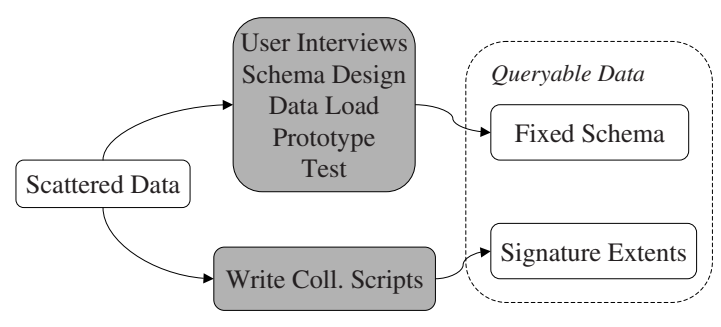

Fig. 2. Two methods of organizing data with different amounts of offline activity. Signature Extents are a form of schema inferred by the system.

\subsection{RDF: Shortest Route to Machine-Processable Metadata}

We have been using the RDF terminology of "subject," "property," and "object." The word "object" connotes a fundamental feature of the RDF data model: Properties can reference arbitrary resources, making the overall data model a graph. This feature distinguishes RDF from tree-based data models, e.g., LDAP [17.

RDF was designed to enable interoperability and automation by adorning web resources with simple machine-processable metadata. Our domain is sim- 
ilar: We adorn files, the substrate of observation and simulation systems, with metadata to enable access.

Formally, an RDF graph is a set of triples of the form $(s, p, o)$. The property (or predicate) $p$ is drawn from a set of Uniform Resource Identifiers $R$. The subject $s$ is drawn from the union of $R$ and a set of blank nodes $B$. The object $o$ is drawn from the union of $R, B$, and a set of literals $L$. Blank nodes act as existentially quantified variables over the domain of a particular graph. RDF graphs can be drawn as a graph using the union of $R, B$, and $L$ as nodes and drawing an edge for every triple. Edges are labeled with the property URI, and non-blank nodes are labeled with either the URI or the string literal in quotes. Blank nodes are, as might be expected, unlabeled.

We forego the use of more advanced features of RDF, such as RDF Schema for defining classes and class membership, and reification for asserting RDF statements about other RDF statements. Use of these features would again require the up-front modeling effort we hope to avoid. We do allow literals to carry a type as supported in RDF. Note that our interest is in the RDF model rather than the XML-based syntax for RDF [16].

Even without the advanced features, the RDF data model can be used to capture the information expressed in much richer data models, though redundancy is often introduced, and query facilities are sacrificed.

\subsection{Interpreting RDF in Our Application}

To model our metadata in RDF, we must first specify the sets $R, B$, and $L$. Construct a set $F$ of URIs corresponding to files in the domain of interest. Construct a set $P$ consisting of properties associated with a file in $F$. The set of URIs $R$ is $F \cup P$. The set of literals $L$ are simply string constants. Collection scripts will only emit triples $(f, p, o)$ where $f \in F, p \in P$, and $o \in L \cup F$.

Figure 3 shows an example of an RDF graph used to model our domain.

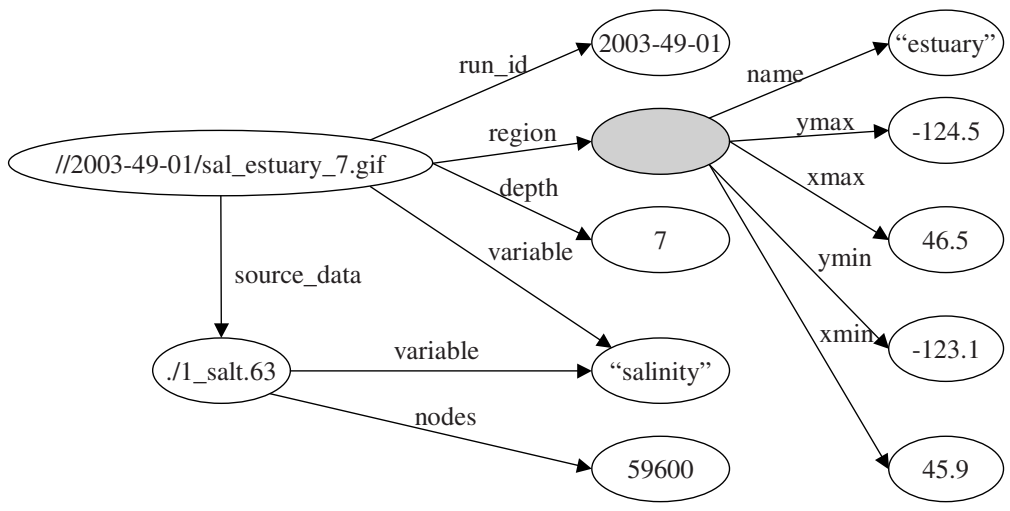

Fig. 3. An example of RDF used to model files and properties in our domain. 
The RDF data model allows a URI to appear as both a property and an object in two triples $x$ and $y$. In our application, however, property URIs may not appear as objects, though file URIs may. Files that reference other files are especially useful for tracking provenance; data products can reference the datasets they are derived from, and datasets can reference the executable of the simulation code used to generate it as well as the inputs.

The graph structure of RDF is one prominent feature RDF; the other is the concept of blank nodes. Blank nodes are used to model anonymous structured types. In Figure [3, we use a blank node (the gray oval) to capture the idea that the region named "estuary" corresponds to the bounding coordinates xmin = 45.9, ymin $=-123.1, \mathrm{xmax}=46.5, \mathrm{ymax}=-124.5$.

By our definitions, collection scripts are not capable of emitting blank nodes directly. To retain simplicity, elements of structured property values are attached to the files directly, a decision that introduces redundancy but retains simple semantics for the collection scripts. In Section 4.4 we will describe how these structured types can be correctly abstracted after extraction and load.

\section{Analyzing RDF Using RDBMS}

We have modeled our domain of files and metadata using RDF. Our use of RDBMS technology to store and manipulate RDF data is the subject of this section. As reported in the literature, RDBMS are an appropriate choice for managing RDF data. However, since we have no schema information at this stage, we adopt a generic schema that captures the RDF triples directly. This approach has been called the vertical representation [3], generic schema, or edge schema [1] in the literature for various data models and applications. We discuss querying the data in terms of SQL: Query languages for RDF have been proposed 1825, but either tend to rely on schema information encoded as RDF Schema [8], or are based on subgraph matching for which scalability has not been demonstrated.

Two variants of the generic RDF schema appear in Figure 4a. A Triples table stores RDF triples in terms of string representations of the URIs. To improve performance, a Resources table might abstract the string representations of uris and leave integer keys in the Triples table for faster processing of set operations on resources (Figure $4 \mathrm{~b}$ ).

\subsection{Performance}

The use of a single table for storing the RDF graph faithfully supports the RDF specification, but makes queries over RDF difficult to write. For example, to retrieve all the files (subjects) which exhibit the properties variable, region, and plottype, 2 self-joins (in standard SQL) are required. Figure 5 shows the SQL for such a query over the schema of Figure 4 a. To ask for files that exhibit an $n$ property signature, an $(n-1)$-way join is required. More generally, each property to be viewed (projection) or used to filter results (selection) contributes a join on 
a)

Triples

\begin{tabular}{|l|l|l|}
\hline subject & property & object \\
\hline $\begin{array}{l}\text { file://forecasts/2003-184/images/anim- } \\
\text { sal_estuary_7.gif }\end{array}$ & property:region & estuary \\
\hline $\begin{array}{l}\text { file://forecasts/2003-184/images/anim- } \\
\text { sal_estuary_7.gif }\end{array}$ & property:variable & salt \\
\hline $\begin{array}{l}\text { file://forecasts/2003-184/images/anim- } \\
\text { sal_estuary_7.gif }\end{array}$ & property:plottype & animation \\
\hline $\begin{array}{l}\text { file://forecasts/2003-184/images/anim- } \\
\text { sal_estuary_7.gif }\end{array}$ & property:source & $\begin{array}{l}\text { file://forecasts/2003- } \\
\text { 184/run/1_salt.63 }\end{array}$ \\
\hline
\end{tabular}

b) Resources

\begin{tabular}{|l|l|}
\hline id & uri \\
\hline 1 & $\begin{array}{l}\text { file://forecasts/2003-184/images/anim- } \\
\text { sal_estuary_7.gif }\end{array}$ \\
\hline 2 & file://forecasts/2003-184/run/1_salt.63 \\
\hline 3 & property:region \\
\hline 4 & property:variable \\
\hline 5 & property:plottype \\
\hline 6 & property:source \\
\hline 7 & estuary \\
\hline 8 & salt \\
\hline 9 & animation \\
\hline
\end{tabular}

Triples

\begin{tabular}{|l|l|l|}
\hline subject & property & object \\
\hline 1 & 3 & 7 \\
\hline 1 & 4 & 8 \\
\hline 1 & 5 & 9 \\
\hline 1 & 6 & 2 \\
\hline
\end{tabular}

Fig. 4. Two possible schemas for modeling RDF in RDBMS.

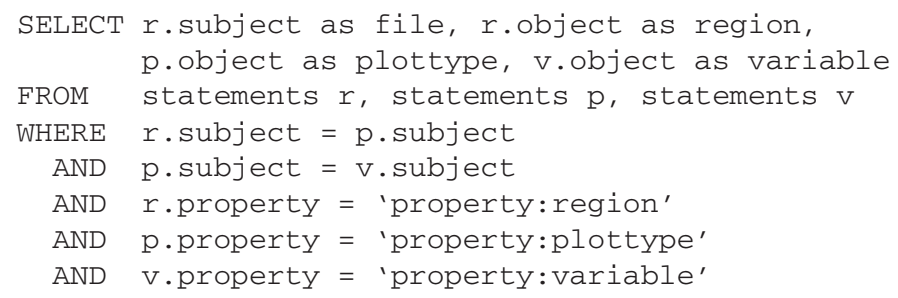

Fig. 5. Simple SQL to find resources exhibiting properties variable, region, and plot type.

the Triples table. The SQL is awkward to generate or write, and performance is terrible with respect to the number of conditions. Our current database captures 30 million RDF triples describing 6 million files, so performance is important.

Using a single Triples table, we can express arbitrary RDF graphs. In practice however, we observe that the files have one of several signatures. For example, most data products derived from simulation output are associated with a variable (e.g., salinity, velocity, temperature), a region (e.g., estuary, far, plume), and plot type (e.g., isolines, transect, timeseries). Figure 6 shows a transect plot (a) and an isolines plot (b) for the salinity variable in the estuary region.

Each of these plots exhibit the three metadata attributes involved in the query of Figure 4. If this signature is common, we can improve performance by materializing the signature extent proactively, reducing the query to "SELECT * FROM <signature name>" 
a)

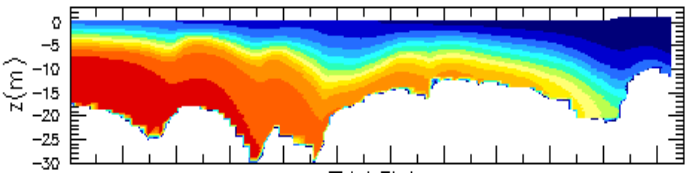

b)

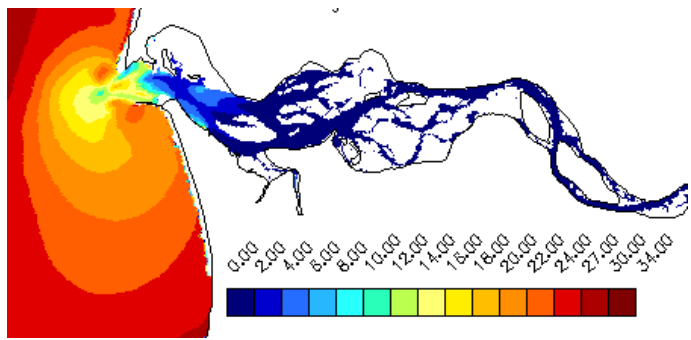

Fig. 6. Two data products from the CORIE simulation system.

\subsection{Signatures}

A signature is the set of properties used to describe a particular file. A signature extent is a set of files that all exhibit the same signature. Computing the extents involves finding the unique signatures exhibited in the current database instance of the Triples table. We materialize these extents as tables in the relational schema. This approach is only wise if the number of signatures is small relative to the number of files. Considering that there are intuitively related sets of files such as data products, simulation outputs, etc., this assumption appears valid. Note, however, that the number of signatures is not simply equal to the number of scripts. Multiple scripts, possibly written by different people, can fire for the same file. Further, nothing constrains a script to output the same properties for every file. For example, we use collection scripts that output different parameters depending on which version of the simulation is being considered.

Computing signatures is similar to extracting association rules in a data mining application. Instead of identifying which item groups tend to be found together in a single customer order, we find properties that tend to be found associated with a single file. However, we need not invoke the full power of data mining, since we are only interested in association rules of 100 percent confidence; data mining algorithms use sophisticated techniques to estimate the confidence level and prune the search space.

Computing the signatures might seem to require application code, but we can exploit set-valued attributes to express the computation using a dialect of SQL. We simulate set-valued attributes in Postgres [30] using sorted arrays. The SQL to compute all the unique signatures in the database is given in Figure 7 The outermost query simply projects out the signatures, removing duplicates. The GROUP BY operator in the nested query applies an aggregation function (array_accum) that constructs an array dynamically from the elements of the group. The result of the nested query is a set of (file, signature) pairs. To model 


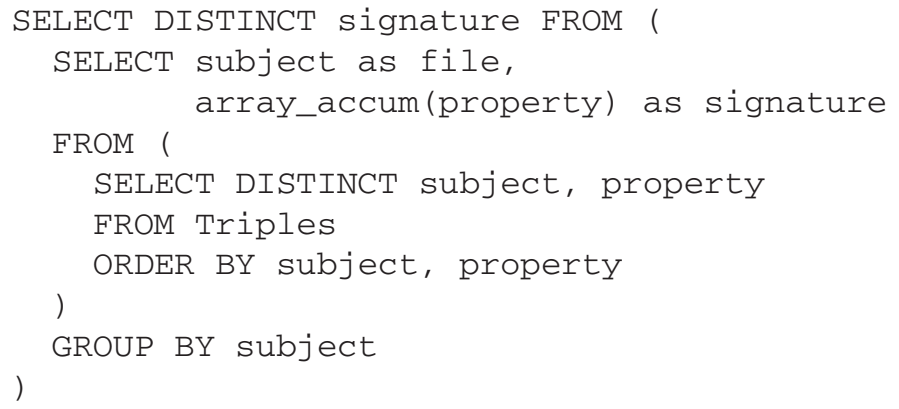

Fig. 7. SQL to compute unique signatures.

sets correctly using arrays, we must remove duplicates and sort the elements; this is the purpose of the innermost query.

For each signature, a signature extent can be computed with attributes for each property in the signature. The materialized view can be computed using SQL of the form in Figure 5, but a much faster method again uses set-valued attributes and a crosstab operation. The crosstab operation is known by various names in the literature. The designers of the SchemaSQL language 20] propose a fold operator, and Online Analytical Processing (OLAP) systems include a CUBE operator [14].

The SQL in Figure 7 can be adjusted slightly to generate the profile for every subject in the triples table (Figure 86) rather than the set of unique signatures. We can materialize this adjusted query, calling the materialized table FileSignatures. Now, for each unique signature $s$, we want to 1 ) find the set of file URIs $F_{s}$ whose signature is $\left.s, 2\right)$ find the set of triples $\left.\left\{(f, p, o) \mid f \in F_{s}\right\}, 3\right)$ sort the results by subject and property 1 , and 4 ) perform the crosstab operation to convert the properties into attribute names in the query results. Steps 1-3 are captured by the SQL in Figure 8b. The result of the crosstab is materialized as a signature extent and is available for further querying by users. This process is repeated for each signature inferred by the system.

This approach is far more efficient than the SQL in Figure 5, since that approach requires $O(n)$ joins, where $n$ is the number of attributes in the signature. The crosstab algorithm is linear with respect to the number of files and the number of triples. Our focus in this paper is on semantics, but our experiments have shown that the improved query is over an order of magnitude faster.

Signatures provide an overview of the data collected with the harvest mechanism. Users can check their assumptions about the metadata that is collected by reviewing the set of signatures found and which signature a particular file exhibits. For example, in our domain, users expected that all files containing parameters to the simulation would have the same signature. However, two similar but distinct signatures were found by the system, exposing the fact that that

\footnotetext{
${ }^{1}$ The single-pass crosstab algorithm used requires that the data be sorted.
} 
a)

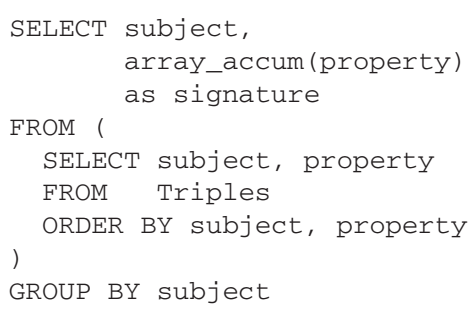

b)

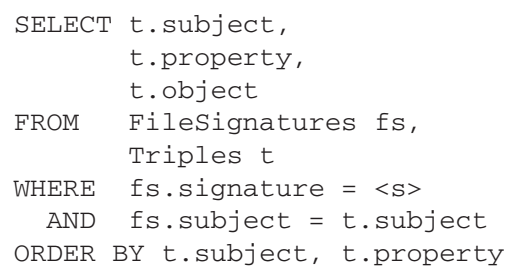

Fig. 8. Partial SQL to compute signature extents. The relation FileSignatures in (b) is the result of query (a).

parameter files created for different versions of the code had a slightly different format. A new collection script to parse the older parameter files was created.

\subsection{Update Semantics}

So far, we have discussed how to efficiently compute signature extents from a large set of RDF triples, but how are these data to be maintained as the RDF stream changes? New simulations result in new files being delivered with potentially new signatures. As requirements change, existing collection scripts will evolve and new collection scripts will be written. New applicability rules may also be defined, causing existing collection scripts to operate on new files. In each of these situations, there are three classes of changes that must be accommodated: new files with potentially new signatures, new properties for existing files resulting in a change of signature, or new values for existing properties of existing files. How the system reacts to these possibilities is the subject of this section.

New Files. When new files are presented to the system, they may or may not exhibit a previously encountered signature. If they do, then a new tuple may be inserted into the appropriate signature extent, and no schema changes are required. If the signature has not been previously encountered, then a new signature extent can be created and a the new tuple inserted into it.

New Properties, Existing Files. If a collection script is modified and then reexecuted over existing data, two possibilities must be addressed. First, the newly modified collection script may emit different values for an existing property of an existing file. We describe this case in the sequel. Second, a collection script may emit an entirely new property for an existing file, changing the file's signature. Since we have previously computed signature extents and materialized them, we must massage the database to accommodate this new signature.

One choice is to simply drop and reload the database whenever the collection scripts are modified or another change is made, but an incremental strategy is 
desirable. If the file's new signature has been previously encountered, then an extent exists for it, and the tuple can be deleted from the old signature extent and inserted into the new one. If the signature has not been previously encountered, then a new signature extent must be created first.

New Values, Existing Properties, Existing Files. To support update of property values, we must modify our interpretation of RDF. The RDF data model allows a single property to be asserted more than once for a particular subject. Instead, we enforce that no two triples in a given database instance may have the same subject and property. To accommodate updates to a property's value, we interpret the assertion of a property as a command to overwrite the previous value, if one exists. This interpretation implies that there is a total order on the RDF triples, corresponding to their arrival in the system.

To define the total order, we must consider executions of the harvest program, executions of collection scripts, and the order of assertions produced by a collection script. Executions of the harvest program can be serialized naturally by the server. However, within a single execution of the harvest program, multiple collection scripts may attempt to assert the same property for the same file. To resolve this ambiguity, we defer to the order of the applicability rules. Recall that these rules specify which collection scripts to fire for which files. The latest rule that causes a duplicate assertion to be made overrides any previous rules. The applicability rules are managed by the data creators just as the collection scripts are, so changes can be made unilaterally. There is also the possibility that a single collection script may assert the same property for the same file twice in a single execution. We rely on the script author to understand that only the later value will be retained.

To minimize redundant work, we keep track of which collection scripts were executed with which execution of the harvest program. If the harvest program is re-executed over existing files, we only fire those collection scripts that are new or have been modified since the last execution. This policy reduces the amount of time required to harvest metadata and reduces the conflicts that must be resolved during loading.

Batches. The semantics we have just described can result in a great deal of data reorganization work. Very small changes to a collection script can result in many insertions, updates, and deletions, and potentially require new database tables to be created. There is a tradeoff between processing new metadata incrementally and buffering batches of metadata to process all at once.

If we enact the policies above after every triple arrives, we will obtain the correct semantics, but we will fire multiple SQL statements for every triple. The volume of data is large enough that this approach is unacceptable. Fortunately, triples generally arrive in batches, usually corresponding to an execution of the harvest program. We interpret the triples in a batch as arriving simultaneously, and only compute the new schemas once per batch. As described, there may be duplicate properties for one file asserted in a single batch. We remove these 
duplicates inside the harvest program, according to the semantics above, prior to the database load operation. Therefore, even though we consider each batch as a simultaneous set of updates, no ambiguity can arise.

In our application there is a natural batching strategy. For each run of the CORIE simulation code, around 20k files and around 100k triples are loaded into the system. If we consider this set a batch, we can balance the tradeoff between stale data and computing signatures that are replaced without ever being accessed.

Deletes. Deletes are more problematic. If we do not support deletes, there is no way to remove properties associated with files to support changing requirements, which prevents the correct signatures from being exhibited. But in order to support deletes, we must allow authors of collection scripts to specify that a file should no longer have a given property. (Note that we do not wish to support deletes of files themselves. The fact that a file once existed is important lineage information.)

We entertained two possible semantics for deletes: 1) Whenever a batch of metadata arrives for a file, assume the old metadata is incorrect and drop it all. These semantics agree with the update semantics already specified; new triples will still "replace" old triples since the old triples are deleted beforehand. The problem with these semantics is that collection scripts are prevented from extending the metadata associated with an existing file. They must produce all metadata for a file in every batch. Another choice is to 2) allocate a special URI "value:delete" that is interpreted as a deletion when it appears as the object of a triple. The problem (other than the inelegance of requiring a specially interpreted value) is that the collection scripts must produce "delete commands" as well as standard metadata, complicating their design and implementation. Although the collection scripts are expected to undergo refinement and change, using them as an interface to perform "one time only" database mutations is awkward. Currently, our implementation uses the second semantics.

\subsection{Blank Nodes Revisited}

Recall that blank nodes represent placeholders for unnamed subjects and objects in an RDF graph. Semantically, blank nodes are existentially quantified variables. In our design, collection scripts can not emit make use of blank nodes. The subject of every RDF triple represents a physical file. Collection scripts have no way of expressing triples whose subject is a blank node.

The disadvantage of this design is that collection scripts must redundantly emit the same information for many files. For example, all region information (xmin, xmax, ymin, ymax, region name) is linked directly to the file rather than linked to a blank node as in Figure 3 The concept of region is a separate entity and should be abstracted using a blank node in the RDF graph. In a relational setting, we note that region name functionally determines xmin, ymin, xmax, and ymax. Normalization procedures prescribe that we decompose a signature extent involving these properties by extracting a "region" table. 
Since the collection scripts cannot express this feature of the data, we rely on tools to discover it after the data has been loaded. Several algorithms exist for discovering these functional dependencies 23. The results of these algorithms can be used to further refine the schema. The tradeoff is twofold: First, a more complex schema may make data exploration more difficult for users unfamiliar with relational principles. Second, the processing of new RDF tuples becomes more expensive as the schema becomes more complex. The logic to transform a batch of RDF triples into a set of tuples becomes complex when considering updates, deletes, and now decomposed tables. Since some changes to collection scripts may require a significant amount of data to be corrected or reloaded, minimizing the complexity of the load operation is important.

For these reasons, we compute functional dependencies and perform prescribed decomposition only offline after a batch of data has been loaded. Further, we publish the data in terms of signatures, even though the underlying schema may be more complex. Modern RDBMS support this form of logical data independence quite well.

Performance is currently adequate considering the database represents over 6 million files and over 30 million RDF triples. After applying the techniques discussed in this paper for speeding up execution of collection scripts, identification of signatures, and population of signatures, we can re-load the entire repository, including re-harvesting the RDF triples, in a few hours. This level of performance is necessary, since there is a possibility that the majority of the database must be effectively reloaded if significant changes to collection scripts are made at one time.

\section{Web Interface}

Having imposed some structure on the metadata stream, we publish the data to the web for validation, exploration, and query. Generally changes to a database schema require changes to an application since applications reference table names and column names explicitly. However, an application can be designed generically by retrieving table names and column names from the catalog rather than hard-coding them. The HTML to display a table's information is generated dynamically from the catalog. We took this approach, extending source code for a free generic database interface [28]. The entry point is a list of tables in the current database.

\subsection{Validation Using Signatures}

The initial view in the web interface shows the signatures inferred by the system. In addition to a system generated name, the properties that make up the signature are displayed, giving an overview of how the files are organized in the database.

The list of signatures computed by the system provides immediate information about what sort of data is being captured. For example, users can see that 
some signatures are very similar, differing in only one property (e.g., <plottype, region, variable, animation> versus <plottype, region, variable>). This configuration suggests that the presence of the animation property is acting as a boolean. If the animation property exists, then the file type is an animated gif; if not, it is a single frame. The value of the animation property may be irrelevant. Perhaps a better configuration is for files in both signature extents to assert a GIFtype property, with either the value animation or single frame. If this configuration improves understanding, appropriate adjustments to the collection scripts can be made, and the database schema will adapt.

\subsection{Query}

The interface also allows users to construct simple queries via the signatures. Properties encountered in the metadata stream are shown, along with a range of values. This kind of summary information is important for validation as well as query formulation. For example, one of the parameters to the simulation, implicitness, indicates a coefficient controlling the weights of implicit and explicit methods for solving for each timestep. Those unfamiliar with the application can see the range of values found in the database before issuing a query. In fact, those unfamiliar with the term use these value ranges as clues to its meaning.

Figure 9 illustrates the query interface. To construct a query, users select signatures from the left, which populates a list of properties on the right. If a single extent is selected, then these properties simply form the SELECT clause of a SQL statement. If multiple signatures are selected, the natural join between the two is computed. Allowing only the natural join limits the expressiveness of this query interface, but has suited our purposes for the application thus far. Defining the precise query features necessary in this domain is an open problem. Selection conditions can be added at the bottom of the screen. The query can also be saved as a materialized view, which is refreshed at system-configurable intervals.

After retrieving the results of queries, the user can view the file (useful for images) or make annotate the file. Figure 10 shows the results of a query, and demonstrates how the user can click a link access the file itself.

The query interface allows us to make efficient use of the scientists' time. We can meet with them for an hour, browse through signature extents, and construct views on the fly. In several of these meetings, we have discovered anomalies in the metadata that reflected real anomalies in the source data. We receive comments such as the following:

- "Why are there only 3 salinity data products for this simulation run? I was expecting 4 thousand!"

- "Why are there two copies of the parameter file for every simulation run?"

- "The date should be divided into month and year; we should change that in the collection scripts." 


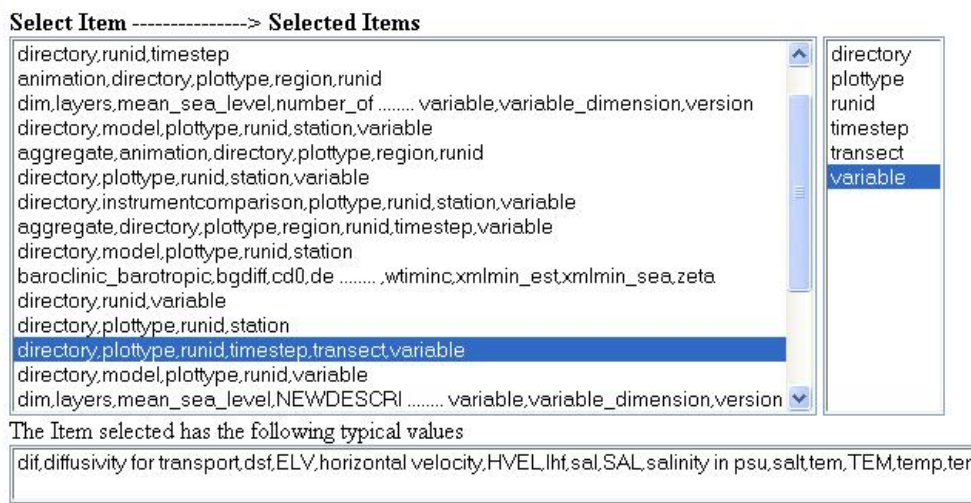

$\square$ Check to include Path

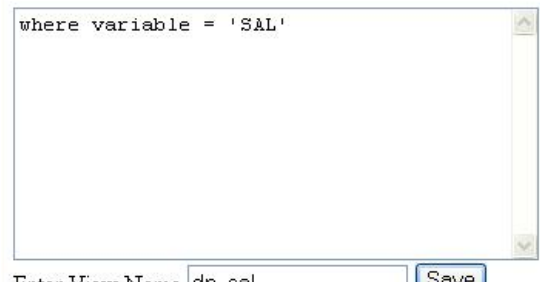

Enter View Name dp_sal Save

Fig. 9. To express queries and saved views, users select signatures on the left and add selection conditions at the bottom.

\section{Related Work}

Our base representation of RDF triples is directly related to the work of Agrawal et al. in managing E-commerce data 3]. Their system also copes with frequently changing attributes by adopting a vertical representation of data that is isomorphic to our base representation of RDF triples. The authors observe that such a representation makes queries difficult to express, as we have also argued. A horizontal view is constructed over the vertical representation of the data to ease this difficulty. The authors' notion of horizontal representation is very different from our signature extents, however. The horizontal representation is a single table that exhibits all attributes exhibited by any item in the database, acting as an implementation of the Universal Relation [22. Null values are used to fill the table where an attribute has not been defined for an item. The resulting table may have thousands of attributes, and many of the tuples are very sparse. Our extents actually partition the data among many "horizontal" tables, each having only a handful of columns and significantly fewer tuples. Using extent tables, we can achieve interactive query performance with at least an order of magnitude more data than Agrawal et al. describe. 


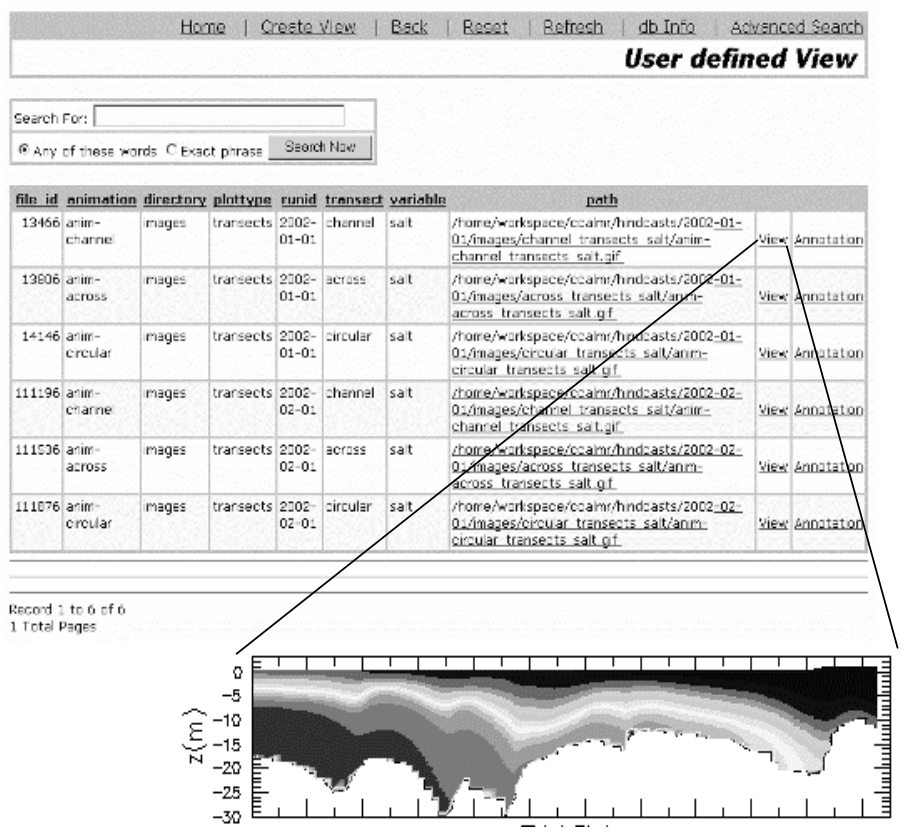

Fig. 10. Query results allow access to the file itself.

Schema extraction from semi-structured data has been studied intensely 12 13:26]. The RDF data model provided a closer match with our desired semantics than the more general semi-structured data models. Further, semi-structured data models are oriented toward graphs with deep and complex structure. The schema information extracted with these systems would not necessarily provide guidance for query formulation in our domain.

RDF databases are gaining popularity in both research and commercial contexts 919 18. These systems organize the data according to RDF Schema information provided along with the RDF data. Contrast this situation with our domain, where the schema information is not recorded explicitly and is frequently changing. Systems that allow querying of unstructured RDF [25] operate via graph matching and have not yet demonstrated scalability. Further, the users are required to "fish" for query results, since no schema structures are present to guide query formulation. Guha has done some early work on a scalable native RDF database that does not rely on RDF Schema, but thus far results are not provided [15].

More recent work by Christophides et al. advocates views over RDF databases constructed as virtual RDF classes [21, which recalls our design. However, users are charged with building the views using a specialized language. We are taking steps towards deriving appropriate views using only the base RDF data and the patterns therein. 
Relational representations of XML data are also of interest. Some depend on RDF Schema information, which we do not have. Earlier work compares generic representations of XML [11]. The edge schema captures parent-child relationships between nodes in the XML tree, and is very similar to the common representation of RDF triples we have adopted. Binary representations involve separate tables for each element label, with results constructed via joins.

Metadata standards such as Dublin Core 1] and those produced by the Federal Geographic Data Committee (FGDC) [2] provide pre-defined schemas for metadata. These standards are usually intended to be human-readable, and are not usually amenable to structured querying. Further, the standards are often intended for large granularity datasets rather than individual files. For example, in our domain, an entire year's worth of simulations may be described by a single FGDC document.

Research on ontology models and tools has become commonplace in the last few years. The OWL languages for capturing ontological properties [6], based on $\mathrm{RDF}$, have become W3C recommendations. While work on ontologies show promise for capturing complex relationships across domains, adoption has been slow due to practical issues. Usually ontology tools assume that a Ontologist, Knowledge Engineer, or Cybrarian [24] is available to model source metadata in the target system. This task is far from trivial, which is why job titles such as those mentioned have been invented. Our framework streamlines the process of converting scattered source data to machine-processable metadata. Inferring and exploiting the complex relationships supported by ontology models remains future work.

The Grid [10] community has recognized the need for a comprehensive metadata management solution in Grid environments. The MCAT metadata information catalog supports metadata processing in the context of the San Diego Super Computing Center's Storage Request Broker (SRB) [5]. More recently, the Metadata Catalog Service (MCS) 29] has been shown to perform well under heavy query workloads and large database sizes 27. Our goals differ from these systems, however. We are working toward a system that can adapt to evolving requirements with little or no user intervention. The MCAT system is designed to process metadata queries over using specialized query structures. While the schemas providing structure to the data are not necessarily fixed, users must explicitly update the schema to reflect changing requirements. The ability for MCAT to handle frequent changes is unclear. Other features such as location transparency and replication are important and complementary to our own work.

\section{Future Work}

There are several research directions to follow based on this work, primarily in the area of finding and exploiting richer structures in the RDF data. Currently we infer simple groupings from the data patterns, and we discussed using functional dependencies to further refine the schema. However, views defined by users in the web interface provide very important information as to how users will access 
their data. Beyond simply materializing these views, the schema can be designed to make these user views efficient. Finding automatic techniques to transform the schema based on user-defined views is an interesting direction to take.

Although we acknowledge that inferred functional dependencies can lead to improved schema design, we have not applied the same analysis to other data dependencies from relational theory: join dependencies and inclusion dependencies. In fact, a rigorous interpretation of these dependencies in our domain would be useful.

We exploit patterns in the metadata stream to facilitate query expression and improve performance. However, a schema serves another purpose as well: enforcement of constraints on the data. If patterns found by the system are acknowledged by users, the system could promote the patterns into hard constraints. After this promotion, violations of the constraints in the metadata stream would be rejected outright as errors. Providing a flexible transition between reactive and proactive pattern exploitation presents interesting theoretical and practical challenges.

The query interface provided via the web supports only simple natural joins, making many useful queries impossible to express. We predict that an interface can be designed that supports all relevant queries in our scientific domain without requiring the full power of SQL.

Acknowledgements. This work was supported by NSF ITR Award No. ACI0121475. We would like to thank the entire CORIE team for their feedback and guidance.

\section{References}

[1] Dublin Core Metadata Standard. http://dublincore.org/index.shtml, 1997.

[2] Content standard for digital geospatial metadata. http://www.fgdc.gov/metadata/metadata.html 2004.

[3] R. Agrawal, A. Somani, and Y. Xu. Storage and querying of e-commerce data. In The VLDB Journal, pages 149-158, 2001.

[4] A. Baptista, M. Wilkin, P. Pearson, P. Turner, M. C., and P. Barrett. Coastal and estuarine forecast systems: A multi-purpose infrastructure for the columbia river. Earth System Monitor, NOAA, 9(3), 1999.

[5] C. Baru, R. Moore, A. Rajasekar, and M. Wan. The SDSC storage resource broker. In Proceedings of the Centers for Advanced Studies Conference, Toronto, Canada, November 1998.

[6] S. Bechhofer, F. van Harmelen, J. Hendler, I. Horrocks, D. L. McGuinness, P. F. Patel-Schneider, and L. A. Stein. Web ontology language reference. W3C Recommendation, http://www.w3.org/TR/2003/CR-owl-ref-20030818/, 1999.

[7] D. Beckett and B. McBride. RDF/XML syntax specification. http://www.w3.org/TR/rdf-syntax-grammar/, 2004.

[8] D. Brickley and R. V. Guha. RDF vocabulary description language 1.0: RDF Schema. http://www.w3.org/TR/rdf-schema/, 2004. 
[9] J. Broekstra, A. Kampman, and F. van Harmelen. Sesame: A generic architecture for storing and querying rdf and rdf schema. In I. Horrocks and J. Hendler, editors, Proceedings of the First Internation Semantic Web Conference, number 2342 in Lecture Notes in Computer Science, pages 54-68. Springer Verlag, July 2002.

[10] A. Chervenak, I. Foster, C. Kesselman, C. Salisbury, and S. Tuecke. The data grid: Towards an architecture for the distributed management and analysis of large scientific datasets. Journal of Network and Computer Applications, 23:187-200, 1999.

[11] D. Florescu and D. Kossmann. Storing and querying xml data using an rdmbs. IEEE Data Engineering Bulletin, 22:27-34, 1999.

[12] M. Garofalakis, A. Gionis, R. Rastogi, S. Seshadri, and K. Shim. XTRACT: a system for extracting document type descriptors from XML documents. In $A C M$ Special Interest Group on Management of Data, pages 165-176, 2000.

[13] R. Goldman and J. Widom. Dataguides: Enabling query formulation and optimization in semistructured databases. In M. Jarke, M. J. Carey, K. R. Dittrich, F. H. Lochovsky, P. Loucopoulos, and M. A. Jeusfeld, editors, Proceedings of 23rd International Conference on Very Large Data Bases, pages 436-445. Morgan Kaufmann, 1997.

[14] J. Gray, A. Bosworth, A. Layman, and H. Pirahesh. Data cube: A relational operator generalizing group-by, crosstab and sub-totals. In ICDE, pages 152-159, 1996.

[15] R. V. Guha. rdfDB : An RDF Database. http://www.guha.com/rdfdb/, 2001.

[16] P. Hayes and B. McBride. Rdf semantics. W3C Recommendation, http://www.w3.org/TR/rdf-mt/, 2003.

[17] I. A. Howes, M. C. Smiths, and G. S. Good. Understanding and deploying LDAP directory services. Technical report, 1999.

[18] G. Karvounarakis, S. Alexaki, V. Christophides, D. Plexousakis, and M. Scholl. RQL: A declarative query language for RDF. In The Eleventh International World Wide Web Conference.

[19] G. Karvounarakis, V. Christophides, D. Plexousakis, and S. Alexaki. Querying RDF descriptions for community web portals. In Journees Bases de Donnees Avancees, pages 133-144, 2001.

[20] L. V. S. Lakshmanan, F. Sadri, and S. N. Subramanian. Schemasql: An extension to SQL for multidatabase interoperability. Database Systems, 26(4):476-519, 2001.

[21] A. Magkanaraki, V. Tannen, V. Christophides, and D. Plexousakis. Viewing the semantic web through RVL lenses. In Second International Semantic Web Conference, pages 20-23, 2003.

[22] D. Maier, J. D. Ullman, and M. Y. Vardi. On the foundations of the Universal Relation Model. ACM Transactions on Database Systems (TODS).

[23] H. Mannila and K.-J. Räihä. Algorithms for inferring functional dependencies from relations. Data and Knowledge Engineering, 12(1):83-99, 1994.

[24] D. L. McGuinness. Conceptual modeling for distributed ontology environments. In Proceedings of The Eighth International Conference on Conceptual Structures, 2000.

[25] L. Miller. RDF Squish query language and Java implementation. Public draft, Institute for Learning and Research Technology, 2001. http://ilrt.org/discovery/2001/02/squish/.

[26] S. Nestorov, S. Abiteboul, and R. Motwani. Extracting schema from semistructured data. In ACM Conference on Knowledge Discovery and Data Mining, pages 295-306, 1998. 
[27] A. Rajasekar. MCAT - a meta information catalog. http://www.npaci.edu/DICE/SRB/mcat.html.

[28] E. Robillard. GenericDB. http://www.genericdb.com/

[29] G. Singh, S. Bharathi, A. Chervenak, E. Deelman, C. Kesselman, M. Mahohar, S. Pail, and L. Pearlman. A metadata catalog service for data intensive applications. In Proceedings of the 2003 ACM/IEEE conference on Supercomputing, November 2003.

[30] M. Stonebraker, L. A. Rowe, and M. Hirohama. The implementation of Postgres. TKDE, 2(1):125-142, 1990. 